\title{
A Cybernetic Participatory Approach for Whole-Systems Modelling and Analysis, with Application to Inclusive Economies
}

This paper was downloaded from TechRxiv (https://www.techrxiv.org).

\section{LICENSE}

CC BY-SA 4.0

SUBMISSION DATE / POSTED DATE

$24-01-2021 / 27-01-2021$

\section{CITATION}

Hassannezhad, Mohammad; Gogarty, Mary; O'Connor, Clementine Hill; Cox, Julian; Meier, Petra S.; Purshouse, Robin C. (2021): A Cybernetic Participatory Approach for Whole-Systems Modelling and Analysis, with Application to Inclusive Economies. TechRxiv. Preprint. https://doi.org/10.36227/techrxiv.13635059.v1

$\mathrm{DOI}$ 


\title{
A Cybernetic Participatory Approach for Whole-Systems Modelling and Analysis, with Application to Inclusive Economies
}

\author{
Mohammad Hassannezhad, Mary Gogarty, Clementine Hill O'Connor, \\ Julian Cox, Petra S. Meier, Robin C. Purshouse
}

\begin{abstract}
Traditional participatory systems modelling demands synchronous time from many experts and face-to-face interaction. This is not always feasible (e.g. recent pandemic) and can restrict which participants can be included. There are additional limitations in the effectiveness of physical paper-based modelling when handling large complex systems with numerous variables and links between them. The key challenge facing practitioners is then how we can retain the benefits of traditional participatory modelling whilst exploiting the advantages of new technologies? This paper contributes to development of an original systematic methodology based on Cybernetic principles. The proposed method, refers to $5 \mathrm{X}$ - standing for Expose, Explore, Exploit, Explain, and Expand, offers a fully virtual co-produced environment for better engagement of stakeholders, developing more confidence in systems mapping, and promote knowledge to other policy areas. A primary application of the proposed method in a real policy setting illustrates its capability in generating a shared policy understanding of complex Inclusive Economy system, where there is conflicting or dispersed knowledge about system structure, refining this understanding through online feedback channels, and transferring this understanding to wider policy and academic partners through mutual relationships.
\end{abstract}

Index Terms - Complex systems; Cybernetics; Fuzzy cognitive mapping; Distributed decision making; Inclusive growth; Network propagation; Online mapping; Participatory systems modelling; Stakeholder engagement; Visual analytics

\section{INTRODUCTION}

E ngaging stakeholders and decision makers as early as possible in the modelling process has increasingly been recognized as a powerful way towards enhancing the credibility of resulting models and leading to better-informed decisionmaking [1]. Participatory systems modelling (PSM) [2] is an increasingly popular approach for stakeholder engagement through development of graphical structuring models - using graphs to express the dependence structure between system variables. It offers a co-produced and co-learning process in which participants with different perceptions, priorities, and levels of expertise work jointly to develop a shared

Mohammad Hassannezhad, the Leading Author, is with the Department of Automatic Control and Systems Engineering, University of Sheffield, UK (m.hassannezhad@sheffield.ac.uk). Mary Gogarty is with Greater Manchester Combined Authority, UK (mary.gogarty@greatermanchester-ca.gov.uk). Clementine Hill O'Connor is with University of Strathclyde, UK (clementine.hill-oconnor@strath.ac.uk). Julian Cox is with the Greater understanding of a system, capture system dynamics under alternative conditions, and recommend policy levers.

In recent years, advancements in information and communication technologies has encouraged academics and practitioners to investigate alternative methods of participation in systems modelling through the exploitation of digital technologies [3]. Traditional methods of PSM [2] bring together groups of people in a fixed place and at a fixed time, using analogue technologies such as flip charts, sticky notes, and marker pens. Despite undeniable capability in encouraging interactivity and exchanging information [4], constraints on both synchronous and face-to-face availability may make traditional PSM less inclusive and limit participation to those who are geographically close. Digital technologies can have positive implications for distributed systems where people are usually located remotely from one another, but also for modelling large complex systems with many stakeholders, many factors and numerous links amongst them. Notwithstanding that digitalization can be an effective solution when physical meetings is not desirable or feasible (e.g., global pandemic; environmental sustainability) while avoiding unnecessary logistics and time commitments [5].

This study was designed to address policy partners' desire to develop a common understanding of the complex system of Inclusive Economies (IncEc) across different groups of stakeholders and policy sectors in their jurisdictions through understanding system drivers and their dynamics. The resultant understanding is intended to facilitate the co-production of science across academic and policy partners (with mutual relationships) in the wider project [6]. This study has also been influenced by disruptions of planned work caused by the Covid19 pandemic and took place under the highly restrictive UK lockdown conditions in March 2020 which demanded the deployment of a fully virtual systems mapping and analysis process.

However, digitalization in the context of this paper goes beyond bringing the participatory workshops online and places

Manchester Combined Authority, UK (julian.cox@greatermanchesterca.gov.uk). Petra Meier is with the Social and Public Health Sciences Unit, University of Glasgow, UK (petra.meier@glasgow.ac.uk). Robin Purshouse is with the Department of Automatic Control and Systems Engineering, University of Sheffield, UK (r.purshouse@sheffield.ac.uk). 
the emphasize on: (1) enabling active participation of stakeholders from the initial stages of systems modelling to post-production analysis and feedback; (2) enabling individual interaction with, and appraisal of, the map to make a better representation of reality by strengthening stakeholders' recognition of the system; and (3) promoting knowledge and reconciling the map to the other policy areas in the long term. Central to this view is the consideration of participatory modelling as a Cybernetic system, that is to say by focusing on information (feedback) channels, interaction of parts, and the structure of systems over time [7].

In this way, we present the development of a systematic methodology referred to $5 \mathrm{X}$ as it offers a dynamic, iterative, and progressive co-learning environment for Exposing the problem complexity, Exploring the system structure, Exploiting stakeholders' understanding of the system, Explaining the findings, and Expanding its application. At the time of writing this paper, the proposed methodology has successfully been run with different sets of stakeholders in different jurisdictions. This paper presents its primary application in a devolved regional UK democracy, the Greater Manchester Combined Authority (GMCA). Technically, $5 \mathrm{X}$ is a hybrid algorithm in the sense that it combines the functionality of conceptual framing methods (mind-mapping) with semi-quantitative mapping methods (fuzzy cognitive mapping) and quantitative network analysis methods (change propagation analysis).

The rest of paper is organized as follows. Section 2 explains the study context and motivations, followed by discussing the theoretical foundation of participatory systemic mapping in Section 3. Section 4 presents the proposed $5 \mathrm{X}$ methodology with application in GMCA. Section 5 discusses insights and learnings from its implementation.

\section{STUDY CONTEXT AND MOTIVATION}

The SIPHER consortium [6] is working closely with three policy partners who work at local, regional and national scales of the UK governance. As part of the SIPHER consortium work IncEc was identified by all three policy partners as a key area of interest and a desire to use participatory modelling to support the development of IncEc policies. The context for this study is GMCA, established in 2011 to facilitate cooperation between 10 Greater Manchester Councils and Mayor, and works with local services, businesses, communities and other partners) to improve the city-region partners [8].

GMCA as an organization focuses on large-scale strategic projects across the city region. A key challenge in GMCA stems from the fact that IncEc goals can only be achieved through well-aligned strategies across different policy areas, including work and employment, education, industrial economy, health and wellbeing, transport, and skills - each with a separate set of priorities and strategies and varying degree of exogenous impact [9]. Defining the exact boundaries of such a system with multiple (conflicting) interests can hence be quite problematic.

Drawing on the analysis of interviews with 8 GMCA officers closely aligned with the IncEc policy priority, it was evident that there was a perceived need for a way to understand the interplay of multiple policies across the system. Interviewees did not use a consistent definition of IncEc and, in some cases, used different language to describe the work they were doing. Nevertheless, there was agreement about the desired outcomes of policy (namely, addressing inequalities) and the need to identify practical ways to deliver these. The different perceptions and definitions of some aspects of the IncEc policy agenda necessitated the need for a participatory approach to systems mapping in order to allow space for discussion and the development of a shared understanding of the system.

Such understanding will then be used to inform (a) academic partners on how policymakers collectively perceive the IncEc system, and (b) policy discussions to shape future decisionmaking. Therefore, it is important that the outcome of this process be maintainable in the longer term (ideally over the cloud to support collaborative activities) as new evidence emerge and more people engage in the process.

\section{PARTICIPATORY SYSTEMIC APPROACH TO POLICY DECISION-MAKING}

Policy decision-making is most effective when all stakeholders (anyone inside or outside the policy system who has an interest (a 'stake') in a particular policy issue) play an active role in the process of problem solving, especially those who are responsible for intended and unintended consequences of decisions [10].

Policymakers typically make decisions based on their perception of a given situation but due to sectoral silos they typically only have detailed insight into their own area of the whole system. Whilst there is often not a direct policy link between the decisions that are made within each of these areas, in practice the decisions taken in one area have consequences that relate to other areas. As such, it is important for policy makers to have a whole-system understanding of how decisions are connected [11].

The participatory process provides a structured knowledge sharing environment so that multiple parties can gain an improved understanding of the reality in form of what drives the system in each policy area and how they are connected to the decisions of other areas [12]. This can eventually enable policymakers to think proactively and make local decisions with a whole-system picture of system boundaries in mind.

At the organizational level, this collective knowledge can be useful for coordinating actions [13][14] and predicting what may happen during a policy implementation process [15][16]. At a more individual level, joint thinking on a given topic can lead to an increase in confidence and quality of decisions [2][5] and greater feeling of ownership [17]. It also makes it possible to identify data gaps and points of synergies and conflicts in views [18][19], hence providing a pathway towards conflict resolution [20][21].

\section{A. Cybernetic view of participatory systems modelling}

From the viewpoint of Cybernetics, IncEc in GMCA is a learning policy system, with characteristics of growth and change, whose structure and behavior are defined by the ways in which system components are interconnected and exchange 


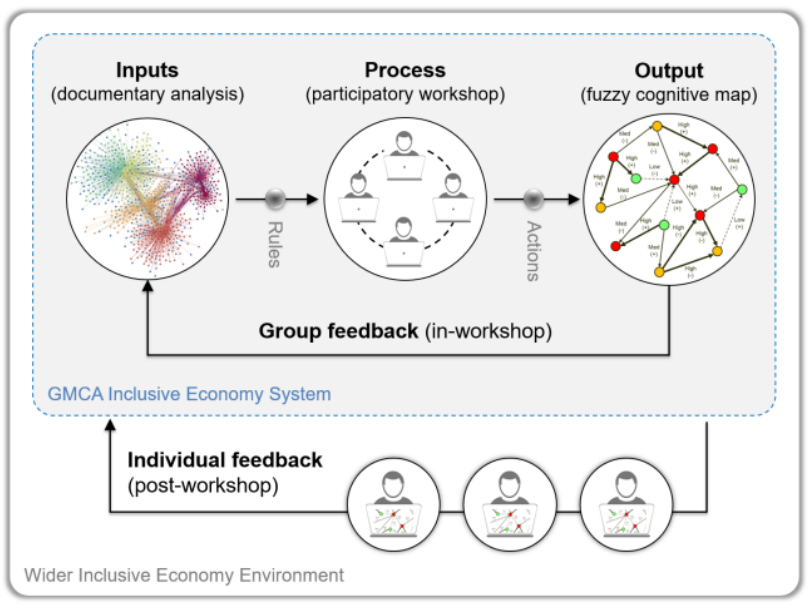

Fig. 1. Cybernetic view of participatory systems modelling

information [22]. The quantity and quality of information is hence central to such systems which can be used for instance to identify new strategies or refreshing existing ones in order to influence the system in desirable ways [23].

The new science of Cybernetics (also called Second-Order Cybernetics [24]) places the emphasis on how 'observers' (i.e., policymakers and experts in our case) construct models of the systems with which they interact. When applied to the context of PSM, this could be a crucial challenge, as the information included in the system being 'modelled' (output in Fig. 1) depends on the nature of information in the system being 'perceived' by participants between which there may or may not be alignment.

Analogous to participatory systems thinking, the core concept of Cybernetics is considering the system as a 'whole' rather than 'parts', yet by highlighting the role of interconnectivity between system components as a mean to understand what should be changed in the system to influence its trajectories in desirable ways [25][26]. The essence of control mechanism in such interconnected systems is identifying an acceptable compromise between positive and negative feedback processes [27][28][29].

Negative feedbacks tend to dampen changes while Positive feedback results in propagating changes with amplifying ('knock-on') effect on the whole system [22]. Quantification of such propagations can enable decision-makers to turn intended positive feedback into negative or vice-versa, also directing unintended consequences towards something manageable where they have the control to either accept or mitigate it [30].

Such quantification would be extremely difficult in organizational settings since the role and influence of people who are making decisions will significantly influence their recognition of system structure. In modelling terms, there are many informal interactions (within and between policy areas) whose propagation will remarkably increase the degree of information channels (i.e., causal pathways) across the system [11], of which the majority are difficult to measure and knowledge of which would be tacit and highly subjective in a participatory process. The next section discusses the factors contributing to effective designing of such systems.

\section{B. Designing a digitalized participatory system}

Successful design and delivery of a PSM require finding a right compromise between several interrelated dimensions. Gray et al. [20] classify them as purpose (the why), process (the how), partnership (the who), and product (the what).

For example, when the problem situation is messy and unclear, conceptual modeling techniques (such as Fuzzy Cognitive Mapping (FCM) [31] and Causal-Loop Diagrams (CLD) [32]) may help to specify the boundaries and bring shared understanding to how the system works. If the purpose is solely to develop a common ground upon stakeholders' knowledge, then this may be sufficient. Alternatively, upon availability of empirical data, such understanding can be used as a baseline for constructing mathematical and simulation models. Examples include transitions from FCM to Agentbased modelling [33][34], from CLD to System Dynamics [35], and from conceptual mappings into Discrete-event simulation [36] and Bayesian belief networks [37] models.

Consequently, the PSM can be seen as an iterative process with decisions made at different stages; for instance about the selection of methods to be used, the choice of inputs and outputs of the process, the inclusion and exclusion criteria for participants (i.e., balancing technical granularity and inclusive participation [38]), and how participants should interact with each other and with the map. In digital format, there will be an additional set of constraints to such decisions; most importantly making sure that participants are able to interact effectively, exchange information over the cloud, have access to the model and underlying data [39], and that facilitators feel confident in using Cloud-based tools.

Such requirements appear to make a distinction between systems mapping practices where in some cases, stakeholders are simply participants of a workshop who interact virtually to draw a systems map but without further engagement with and ownership of the outcome model. For example, Wilkerson et al. [4] recently shared their experience of delivering a fully virtual workshop in an environment context. Apart from limited capability of the tool in enabling participants to visually interact with each other and add information to the links, post-workshop analytical capabilities, validation, and future use of the constructed map seem very limited which requires significant time and ongoing efforts from modelers. If designed effectively, digitally-enabled models can overcome such limitations by providing policymakers the opportunity to individually interrogate and comment on the map with respect to their own area of the whole system, thus leading to a more refined representation of the system before any possible further workshopping. This is particularly important over time, where data evolves or an external factor influences the whole system, the outcome systems map be maintainable and scalable beyond the near-term project, for example through a 'live continuous support' on the web [2]. 
In view of that, there has been growing attention in the scientific community for the development and (or) deployment of web-based software tools that are capable of eliciting, populating, and analyzing data in real-time. The development of spatial agent-based simulations has been a particular focus of existing studies [5]. Two emerging concepts are Active Citizenship (the use of GIS technology within public participation) and Serious Games (computer games primarily designed for a functional purpose rather than pure entertainment), supported by tools such as NetLogo [40], SeaSketch [41], and GAMA [42].

In this study, FCM is the preferred choice for developing a systems map based on group of experts' knowledge. That is chiefly because it is relatively quick and easy to populate and parameterize from varied sources of qualitative knowledge [43] with flexibility in representation (as more components are added to the system) [44], modest time investment, and a degree of transparency to non-technical experts [22, Table 1]. Comparing to conceptual mapping techniques such as CLD, FCM allows more resolution on the nature of links [45] which can be used to quantify and analyze structural dynamics of the system across individual or groups of participants [46]. Comparing to quantitative participatory techniques such as agent-based simulations and system dynamics, it does not require a lot of empirical data and explicit systems knowledge [22, Table 2] - thus making FCMs suitable for data-poor and multi-interest situations [47].

\section{Tools for Fuzzy cognitive mapping}

FCM is a class of Signed Fuzzy Weighted graphs, and typically include feedback loops (reflecting circular patterns of causation) and consisting of nodes (indicating descriptive components) and directed edges (indicating cause-effect relations) between them [48]. It offers a systematic and intuitive approach to combine the experiences and expertise of individuals with various qualitative knowledge [47]. This is crucial in distributed systems like IncEc where local knowledge plays a vital role in capturing a whole-system understanding.

FCM is a static model. While it cannot reflect temporal and spatial dynamics [49], its semi-quantitative representation of cause-effect relationships - typically bounded in a normalized range of $[-1,+1]$ - allows the model to simulate 'what-if' scenarios to understand the impact of different policy options. In this sense, FCM is a dynamic modelling tool whose further iterations (e.g., through individual feedback in Fig. 1) can eventually provide a better resolution of the system structure [44]. Advancements in FCM learning has demonstrated acceptable capability in applying a range of network metrics [49] to quantify the amplifying impact of negative and positive feedback loops - typically referred to network propagation [30]. This would make it a suitable method for designing a Cybernetic participatory system.

There are many software tools (both open-source and commercial) for modelling and analysis of FCMs [50]. Examples include FCM designer [51], Mental Modeler [43], OCAM (Online Cognitive Automatic Mapper) [52], FCM
Expert [53], Cofluences [54], and the commercial Kumu® Amongst them, Mental Modeler and Kumu currently both allow web-based collaborative modelling and limited network analysis but comparing to the former, Kumu represents better functionality for modelling large complex maps (with many nodes and numerous links) with flexibility in tagging data to nodes and links, displaying intended causal pathways, and making separate comments (and associating them to the point in the map), which can be used and maintained by individual users after the workshop. Therefore, Kumu was the preferred option for implementing the methods developed in the present study.

\section{THE PROPOSED 5X METHOD}

This section describes a systematic participatory methodology for policy systems modelling and analysis. The proposed framework expands the paradigm from traditional PM methods (focusing on the physical paper-based workshops) to enable stakeholders to fully (and virtually) engage in the process during pre-workshop design (aligning mapping purposes with stakeholders' needs), in-workshop elicitation (identifying system drivers and their pathways), and postworkshop evaluation and feedback (identifying gaps and change priorities), in an iterative manner. The ultimate objectives are threefold: improving engagement, system identification, and insights into dynamics of the system.

Technically, $5 \mathrm{X}$ is a mixed-method in the sense that it combines the functionality of conceptual framing methods (mind mapping and interviews) with semi-quantitative mapping methods (FCM) and quantitative network analysis methods (change propagation analysis).

In addition to being a fully virtual mapping process, the proposed methodology provides new methods for enhancing the efficiency of mapping workshops (through live polling and prioritization scheme), for amalgamating multiple maps without simplifying the original ones - in contrast to the common approach in the literature [47][46] - and for designing cybernetic participatory systems (through enabling individual feedback channels and promoting learnings to other policy areas). These $5 \mathrm{X}$, illustrated in Fig. 2, are presented in the following in the context of GMCA IncEc system.

\section{A. Exposing problem complexity}

The first stage is concerned with broadening our general knowledge of the problem (in terms of purpose, scope, boundaries) and translating this understanding into a set of requirements for the mapping process. Such understanding will then be used to design a more effective process and making up an effective team of stakeholders. In doing this, there are some principles that should be taken into consideration in advance:

Principle 1. Balancing an adequate representation of the breadth of the system against the depth of granularity of information required by the heterogeneous users of the map. The collated evidence obtained from interviews, documentary analysis, and discussions within the core development team (including people from both academia and policy partner) 


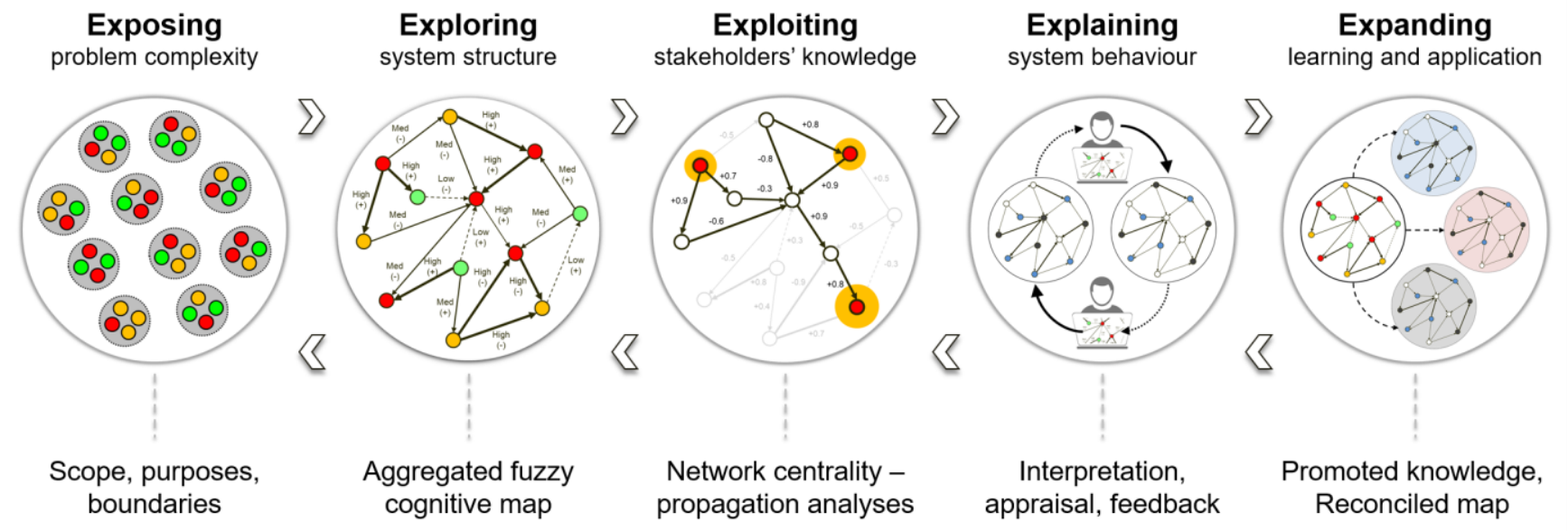

Fig. 2. Overview of the proposed 5X: an iterative and progressive methodology for cybernetic participatory systems mapping

highlighted the multi-interest and complex nature of the context, i.e., understanding the factors influencing successful delivery of IncEc very much depends on who you are talking to, i.e., their role, experiences, and aspirations. Furthermore, there is not a single 'right' level of granularity from which a whole-system problem can be mapped and analyzed. As a result, the core development team defined the key goal as understanding improvement levers and change priorities in the system, based on stakeholders' knowledge. The specific objectives, in particular, are:

- Building a model informed by collated evidence: identifying, mapping, and articulating the key performance indicators and the likely causal pathways between them;

- Building consensus for policy decision-making: consolidating knowledge and creating a shared representation of reality by expressing beliefs; and,

- Building trust in the model and its outputs: Engaging participants actively in the modelling process, increasing confidence in understanding, using, and appraising the model. This will eventually enable academic partners understand how policy partners perceive the system, thus helping them to develop simulation models (out of the systems map) that are more relevant and trusted.

Principle 2. Balancing the complexity of the system (perceived reality) with the complexity of its abstraction model (systems map). That is to say, it is vital to narrow the scope of the modelling in order for it to be feasible and manageable in an online setting, whilst also getting a sufficient resolution of the system to understand key drivers and underlying causal pathways. An online mind-mapping tool was used (and iterated within the core development team) to get an impression of the boundaries of the IncEc system in GMCA and identifying the right settings, in terms of the time and resource required for the workshops.

Principle 3. Purposeful selection of workshop participants, balancing the diversity, breadth and depth of knowledge. In our case, for example, we needed a combination of people with good whole-system knowledge - working at the intersection of a range of policy area - and others with more specific deeper expertise in one or two area(s). Further consideration should be made on finding the right balance between getting most relevant individuals (to the scope of the workshop), their resource and time commitments, and their expectations.

Key activities include agreeing an invitation list, assigning participants to workshop groups (for breakout discussions), identifying roles and responsibilities, choosing the right set of facilitators and preparing them (through collectively agreeing how facilitation will work), creating a guide, script, and prompts for workshop sessions. The output of the first stage is expected to be a detailed workshop plan with an agreed list of participants and facilitators, and a baseline map - acting as the kick-off point of conversations in the workshop.

In developing the baseline map, the overarching GM Strategic Plan, the Greater Manchester Strategy [55] was used as the reference point, since all selected participants were aware of it - and it sets out the key priorities for Greater Manchester over the next five years. It was an iterative process, starting with 58 components extracted from GM Strategy plan (across five policy areas) and 349 components (across eight domains) from other policy documents; this was then merged with the outcome of individual interviews with policy officers (resulting an additional set of 19 components) to conceptualize the baseline map. This map, containing all important priorities and outcomes in GMCA based on agreed view, acts as the starting point of conversation in the workshop sessions, instead of starting from scratch. Whilst this was seen to be a more engaging process as compared to starting with a blank page, we would suggest to keep it as simple (and familiar to participants) as possible, i.e., the GM Strategic Plan in our case, to encourage contributions during the workshop. 


\section{B. Exploring system structure}

The key objective of the second stage in $5 \mathrm{X}$ is making sure that all the important variables of a system and the perceived causal relationships between them are elicited from the participatory process. Causal relations may contain various forms and levels of information, typically capturing in some way that an increase or decrease in one variable causes an increase or decrease in another [56]. These relations can be represented in the form of linguistics (e.g., "low", "medium", "high") or probability values (within the interval of $[0,1]$ ) or even a mathematical function, subject to the availability of reliable empirical data.

A prominent challenge in developing FCMs in this study is that the IncEc system comprises numerous concepts (i.e., nodes in FCM), that relate to different policy areas and can infer different meanings, of which the majority can be recognized as 'important' to a certain extent. Capturing all these important nodes by groups of policymakers and reaching consensus is highly challenging, given they usually have very limited time available. Therefore, we propose an approach to collate individual views by engaging participants in the design process before the workshop, through designing an online polling system. This subsequently leads us to the development of a prioritization system to effectively manage activities during the workshop sessions.

An online polling system. The GM Strategy describes a set of 10 interdependent Priorities for the period of 2019-2024 [55]. Each priority in the document is associated with a range of Strategies that drives the system to achieve a set of (overlapping) Outcomes. The primary discussions with key partners showed that the importance of these components would potentially be different for different policy areas (Fig. 3). For example, 'Northern Powerhouse Strategy' may be an essential strategy for business and economy sub-systems but may be less important strategy for education and health. Besides, participants' limited understanding of the importance of drivers and outcomes in other policy areas may affect their engagement as well as the resulting map.

In response, we extracted the information from the GM Strategy into a set of questions, aiming to understand the relative importance of system components based on a five-point Likert scale, ranging from "Not important at all" to "Modest importance", "Important", "Very important", and "Essential". A live polling system was designed and implemented in Slido® and communicated with the confirmed participants (together with an information pack) a few days before the workshop. The benefit was twofold for the development team: enabling each participant to think globally and getting a broader view of what drives the IncEc system in other policy areas - by bringing inputs from other policy areas between which there may be no direct communication; and enabling us to design the workshop sessions effectively by focusing on the most important components, based on an aggregated view of participants.

A prioritization system. Traditionally, participatory workshops are organized for a full-day (or sometimes two consecutive days). When engaging senior policymakers, it would be very difficult to bring a representative group together for more than few hours of a single day - even with a long notice period. To run an effective workshop while taking this limited availability into consideration, we propose designing a prioritization mechanism to start the workshop session with areas that participants previously recognized as more important. That would only be applicable if we can get an initial impression from participants in advance. Assuming the IncEc system as a multi-layer network of Priorities, Strategies, and Outcomes, prioritization can take place at two levels:

(1) At the system level: we focus on specific classes of components and their causal pathways. With three layers of components in the system, the core development team identified four levels of priority to manage the workshop conversations (Fig. 3), respectively:

- P1: mutual links (interactions) between outcomes;

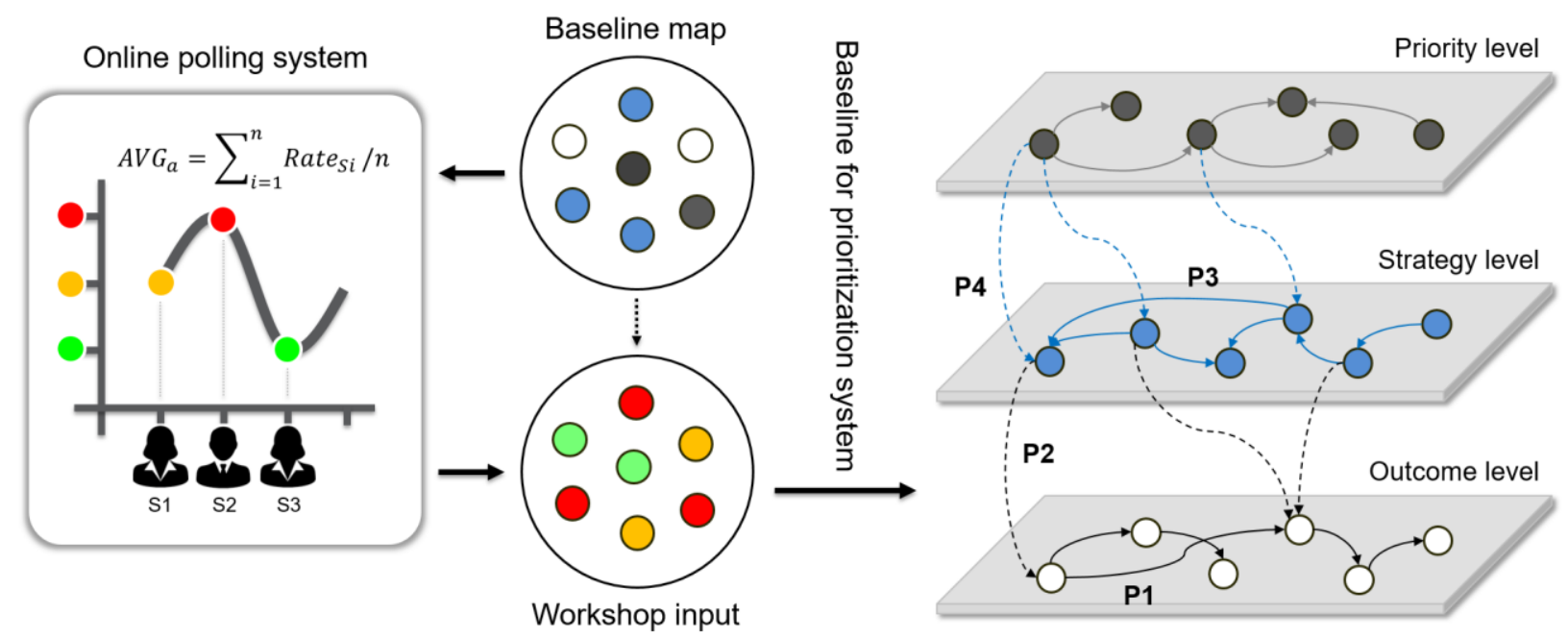

Fig. 3. Designing an online polling system (where each participant voted for importance of components) and a prioritization system for workshop 
- P2: impact of strategies on outcomes;

- P3: mutual links between strategies; and,

- P4: mutual links between priorities and strategies.

(2) At the component level: within each priority level, we use the aggregated response from the online poll and progressively discuss the Essential components and then Very important and Important components, respectively. These components are distinguished by color-coding in the interactive mapping platform in Kumu (Fig. 4).

The advantage of a prioritization system is that, within a limited time-frame, it ensures the outcome map will minimally represent all important phenomena captured collectively by participants. A disadvantage is that the collective importance of components may not necessarily be aligned with the individual view of some participants, thus affecting their degree of engagement. However, when refining the outcome map, there will be sufficient breadth for participants to reflect on their views and share these with others in a considered manner.

Designing scripts and prompts. In traditional physical workshops, the facilitator role principally involves taking care of mutual learning and active engagement to help foster a collaborative environment [21]. In virtual settings, however, the role of coaching and facilitation becomes more complex, as facilitators have to also interact with the online mapping tool and make information visible in parallel to the aforementioned tasks. Aligned with the literature [57], we found it would be more effective to use two facilitators per group, one for interacting with and translating data into the mapping platform and the other for encouraging interactions and managing the discussion, plus a coach with a floating role across groups and taking care of technical (unexpected) issues, inquiries, and the flow of breakout sessions considering workshop purposes.

To reflect the prioritization system in the mapping platform while supporting facilitators in running workshop sessions effectively, a range of scripts and prompts were designed into the mapping platform. Furthermore, a mock workshop was organized, including a training session managed with the core development team and facilitators to make sure they feel confident in interacting with the tool and that they have a clear understanding of the workshop process, in terms of input attributes (information needs to be obtained at each session), expected output (the FCM model), and the prioritization system. The input attributes visualized in the baseline map in form of color-coded nodes and links, as per shown in Supplementary 1.

Aggregating systems maps. The output of our participatory workshop was four FCM models (obtained from four groups) with each allowing free text qualifiers around components; these provides extra piece of information on each component which could be used as part of the aggregation process. These elicited maps, with details provided in Supplementary 2, need to be aggregated into a unified map for learning and analysis. The literature usually treats it by condensing the maps either qualitatively (e.g., by combining the concepts and nested into the upper level [47]) or quantitatively (e.g., by applying the concept of near-decomposability [58]). In either ways, aggregation is a mean of simplification that results into losing information.

In this study, we introduce a set of metrics for aggregating multiple maps. Central to such metrics is examining the input attributes of links that each group added to their local map during the workshop, and quantifying the consensus on the nodes and links, by understanding:

- Contribution (Contr) of a link: how many groups agreed there is a link between a pair of components? For example of a given link between Component $a$ and Component $b$, Contribution $_{a b}=\{B C\}$ determines that two groups of $\mathrm{B}$ and $\mathrm{C}$ (out of four groups) identified this link in the workshop breakout sessions.

- Collective Importance ( $\mathrm{CIm}$ ) of a link: quantifies the rate of Contribution of a link, based on the total number of groups with the assumption that all groups are equally valid with the same weight. In the given example above, if Contribution $\left.{ }_{a b}=\{B C\} \rightarrow \operatorname{CIm}_{A B}=2 / 4=0.5\right)$.

- Collective strength ( $\mathrm{CSr}$ ) of a link: represents the proportional probabilistic strength that the Contributed groups assigned to a link (in terms of strong, moderate, or weak). The popular Weighted Geometric Mean method is applied for this purpose, by following (1), where $k$ refers to the number of Contributed groups (maximum $m=4$ here) and $\lambda_{k}$ shows the weight of each group if they differ. In the above example, $\lambda_{2}=0.5$, as two groups out of four are contributed to this link with equal weights.

$C S r_{a b}^{(c)}=\prod_{k=1}^{m}\left(\text { Strength } h_{a b}^{(k)}\right)^{\lambda_{k}}$

The above formula does not support negative numbers, so for aggregating multiple probabilistic values with the range of $[-1,+1]$, we would suggest applying a normalization algorithm such as Feature Scaling [11] to restrict the values between $[0,1]$.

- Collective Confidence $(C C)$ of a link: is a proportional value showing how many groups were sure that there was a link between components. This is a probabilistic function of Contribution (representing how many groups) and Confidence (representing if those groups were Sure about the link). Therefore, the Least Confident scenario is when there is only one group that identified the link and that group is not sure in it; the Most Confident scenario is when all four groups agree that there is a link and all are sure in it. Details of computing the value of $C C$ is presented in Supplementary 3.

The output of this stage is an amalgamated map that shows the collective view of stakeholders and also the agreed (critical) view of stakeholders (Fig. 4) - helping to identify the synergies and conflicting views between groups. For example, based on the measure of Collective Confidence $(C C)$, the aggregated FCM can be filtered down to visualize the Most Confident view $(C C \geq 0.8)$ - meaning that at least three (out of four) groups agreed there is a link and feel 'Sure' about it, Moderate Confidence view $(0.5 \leq C C<0.8)$ and Least Confidence view $(C C \leq 0.4)$. 


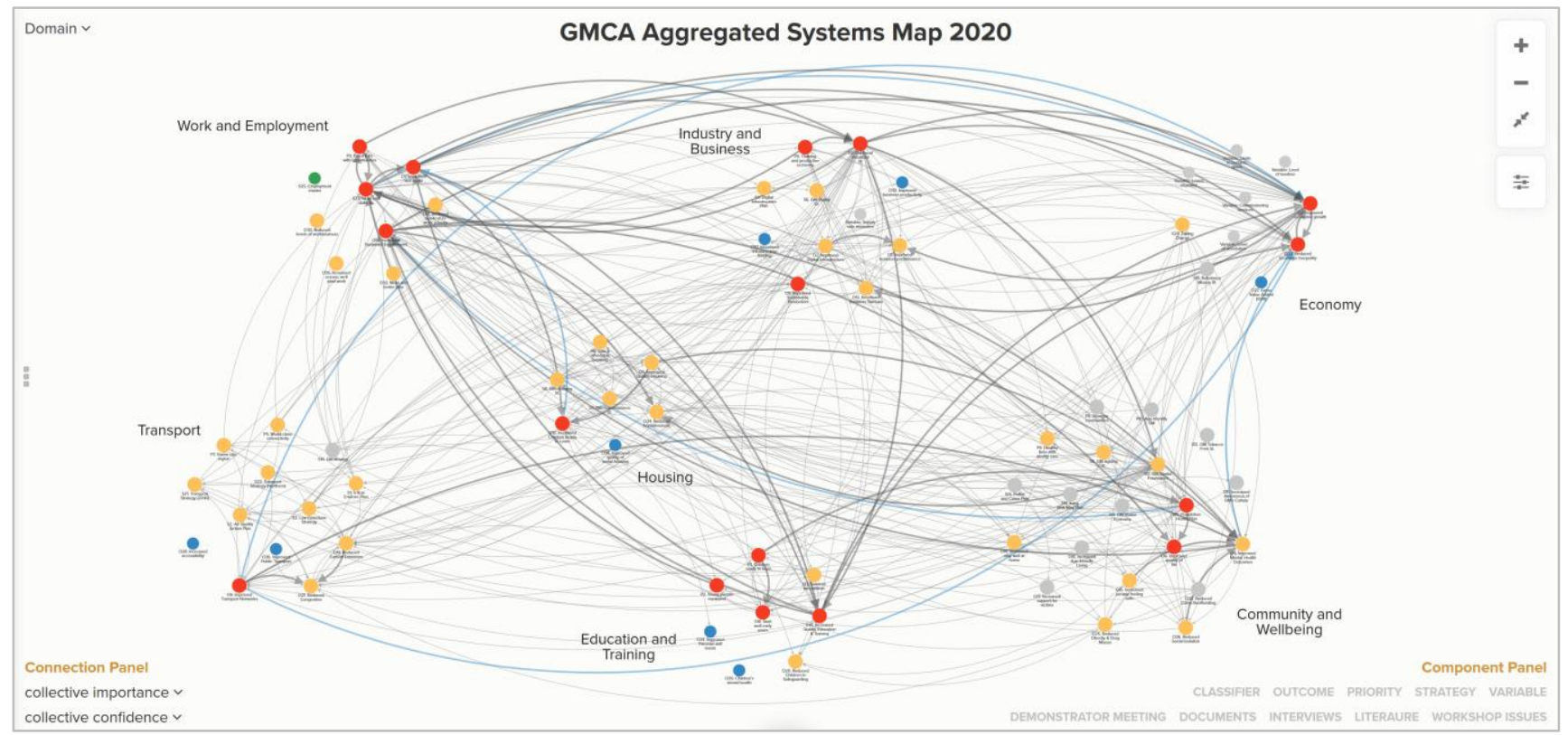

Fig. 4. The amalgamated map showing the collective view of participatory workshop across seven IncEco domains; Control panel: enabling user to customize the view based on system domain (top-left); Collective Importance, Collective Confidence of links, and Contribution of groups (bottom-left); and Class of components and their source (bottom-right). Color-coding components: Red (essential), Yellow (very important), Grey (important), Green (strategy, regardless of importance), and Blue (outcome, regardless of importance)

\section{Exploiting stakeholders' understanding}

The amalgamated FCM model represents the collective thinking of participants, which can be used as a new baseline for interrogation, calibration, and gaining insights. The literature typically move this forward by arranging followingup rounds of workshopping with (the same or a different group of) stakeholders [44] in which they work around limited business scenarios (narrowing down the scope) or refining the original map (broadening the scope) [31].

However, between the first round of workshopping and further iterations (aiming to getting a better resolution of the system), there is typically a gap of individual interrogation and evaluation of the map [47]. This makes more sense in policymaking as it is quite likely that every policymaker would come up with numerous scenarios equally important. Furthermore, in situations where it is not possible to conduct a second round of workshops with the same set of participants, there is a risk of repetition without finding resolutions - because of different perceptions and aspirations.

The proposed framework aims to fill this gap by enabling stakeholders to interact individually with the aggregated map, while developing the analytical capabilities to enhance their understanding of the whole system, and interrogate its dynamic properties from their own knowledge (or expectations) of the system. The core of such capabilities is designing an interactive mapping platform that is equipped with real-time network analytics. Using the semi-quantitative aggregated FCM model as the baseline, we use Network Centrality metrics to identify the criticality and changeability of components and complement it by incorporating the concept of Change Propagation Analysis [30] in order to quantify dynamics of changes and their 'knock-on' effects (resulting from propagation of positive feedback loops).

Network centrality analysis. In network science, centrality metrics indicate the importance (of system components) as intermediaries in the flow of information and materials through the system. Applying such metrics to the aggregated FCM model, illustrated in Supplementary 4, suggest the most influential drivers of IncEc in GMCA as:

- GM Local industrial strategy (S9),

- GM Spatial framework (S11) and,

- GM Work and skills strategy (S23);

and the most influenced outcomes of those strategies are:

- Improved economic growth (O3),

- Increased sustained employment (O18) and,

- Reduced economic inequality (O23).

When defining the prioritization system, a forwarding flow of information was assumed from Priorities to Strategies and from Strategies to Outcomes. Therefore, it is not surprising that most of Strategies in Supplementary 4 are found to have a higher level of activity (out-degree over in-degree) and Outcomes with more passivity (in-degree over out-degree) in the network. By looking at the backward flow (feedback loops), these outcomes in turn have the most effect on these Priorities:

- $\quad$ Good jobs with opportunities (P3);

- Thriving and productive economy (P4); and,

- Healthy lives with quality care (P9).

The network analysis (Supplementary 2) also specifies the GM Moving strategy (S10) and Increased age-friendly living strategy (O10) as transmitters of systems change (with positive out-degree and zero in-degree) and the Increased support for 
victims $(\mathrm{O} 17)$ and Reduced children in safeguarding $(\mathrm{O} 20)$ as receivers of change (with positive in-degree and zero outdegree). In both cases, it would be beneficial to understand the rationale for transmitters and receivers, i.e., it is possibly due to time limitation in the workshop, so post-production individual interrogation of the map may help to get additional important links.

Network propagation analysis. It was previously mentioned (in Sec. 3.1) that quantifying the amplifying effect of positive feedback loops is the essence of control in cybernetic participatory systems, because of their (concurrent) propagation across policy areas via hidden (intuitive) links. Understanding of such 'hidden influentials' [30] requires more sophisticated algorithms (than centrality metrics) to quantify the indirect effect of propagations. We approach this challenge by translating the aggregated FCM model into a change model using the Change Prediction Method (CPM) [59] algorithm implemented in the Cambridge Advanced Modeller [60], where the algorithm uses the information about Collective Confidence (the likelihood of propagating a change) and Collective Strength (as the impact of propagation) to produce the measure of risk.

The main outcome of CPM would be a risk portfolio plot showing different types of change propagation behavior (Supplementary 5A). According to the results, the components S23, S9, and S16 are found as multipliers (bottom-right quadrant) with high influence on the rest of system but hardly affected by them. The top-left quadrant shows the components O23, O3, and $\mathrm{O} 18$ as absorbers; they have a small effect on the rest of system but have a high risk of being influenced by changes to other components - just reaffirming our previous findings in Supplementary 4. At a more detailed level, the risk plot (Supplementary 5B) shows that a change in for instance S23 have highest influence on $\mathrm{O} 23, \mathrm{O} 3$, and $\mathrm{O} 7$ respectively, and on $\mathrm{O} 14$ and $\mathrm{O} 18$ at a lower level.

This sort of whole-system level inference would help policymakers to predict unintended consequences of a decision or at least mitigate its impact to a certain extent. This is particularly valuable when there seems not to be a direct dependency between components. For example, although the aggregated FCM model shows no direct effect from improved sustainable production $(\mathrm{O} 8)$ to reducing economic inequality $(\mathrm{O} 23)$, our propagation path analysis illustrated in Supplementary 5C reveals that there are multiple ways this could happen in practice, through their interfacing components $\mathrm{O} 3$ and $\mathrm{O} 18$.

The information obtained from applying network centrality and propagation analyses can be embedded into the interactive mapping platform to support the user with a dynamic visualization of different views of the system. A color-coding scheme is designed for this purpose in that size of components indicates their importance from a network perspective (based on in-degree, out-degree, and betweenness in multiple views), color of components indicates their importance from participants' perspective (essential in Red, very important in Yellow, and important in Grey), and thickness of links show their importance (in terms of Collective Strength and Compound Risk of propagation).

\section{Explaining system behavior}

The outcome of previous stage is an interactive systems map that is equipped with network analytics (showing three parallel views - Supplementary 6) and dynamic visualization. It shows the current static behavior of the system, under the given structure, which needs to be shared with stakeholders and tested under various structural settings. The focus of this stage is twofold: (1) extending the implications of the systems map conceived by GMCA policy partners to other audiences (e.g., other policy partners, academic partners) of the model (not necessarily participants of the workshop) and understanding their needs. There might be different interests for example in the process, in outcome map, in quantitative aggregation method, or in qualitative social learnings; (2) Designing an effective way to communicate system behavior and getting feedback. Careful consideration of such needs is required to ensure ongoing engagement with stakeholders while enhancing the utility of systems map.

In multi-partner projects such as the SIPHER consortium, policy partners are not the only stakeholders of systems map and our academic partners will be using it as input to build up dynamic simulation-based models of IncEc. We approached this by arranging a series of virtual map-sharing meetings that gave an overview of the process, shared the initial findings, and demonstrated the interactive platform, together with instructions for submitting structured feedback.

On the partner side, through a follow-up communication with representatives, all this information was collated into a postworkshop information package and communicated with participants of the workshop together with an individual (and secured) access to the online mapping platform and also access code to online polling system to share their reflections on the initial findings. The outcome will then determine the necessity and properties of the next iteration of workshopping, e.g., going back to stage 1 (discussing about new purposes, granularity and scope), or to stage 3 (making clarifications on learnings. We believe this complementary approach - group model building and individual map testing - enables stakeholders to identify what is missing or should be modified in the model to make it a better representation of the reality, while overcoming some of the limitations facing the individual-level verification of FCM models in the literature [47].

\section{E. Expanding application and learning}

As mentioned earlier in Sec. 2, the SIPHER consortium is working closely with three policy partners who represent local, regional and national scales of the UK policy government [6]. While each partner is working on its own priorities and strategies to develop whole-system mapping of IncEc system, our primary study shows some degree of overlap in their boundaries and practices. This raises prospects about the extent to which the learnings from applying the current mapping 
process can be expanded to be used in future systems mapping practices with other partners.

Beyond that, complex policy systems such as IncEc involves many concepts from a range of interconnected policy areas such as environment and transport, education and employment, and health and wellbeing. We believe expansion can also happen at the component-level and existing information be used in mental modelling of other policy areas (such as health and wellbeing). This can lead to a major contribution to the scientific literature of participatory mapping - where there is currently a sensible gap [21] - to develop a scalable mapping system that promotes knowledge and learning and reconcile the map to other policy areas.

At the first instance, and after delivering three workshops with all policy partners, the data from three FCM models are collated to build a master database. This database, containing detailed information on drivers of IncEc, their outcomes, and their underlying links, will be used as a reference point in any future systems mapping activity while being used by academic partners in the wider project - thus adding to the credibility of proposed mapping process and its outcome maps.

\section{LESSONS FROM IMPLEMENTATION}

The design and delivery of a fully virtual systems mapping process required a co-production process with policy partners. This was undertaken over a period of ten months (starting in Oct 2019), with the first three months spent on interviews, and then the implementation of each stage respectively at month five (exposing), six (exploring), eight (exploiting), eight-ten (explaining) and ten-onwards (expanding).

This study brought both methodological and implementation novelty to the field by pushing the boundaries of IncEc participatory modelling practice to online version with considerations to the theoretical enrichment by focusing on the way that data are elicited, aggregated, analyzed, and refined in short- and longer-terms. Based on the feedback acquired from our participants, facilitators, and the core team, we would highlight few learning points in the following:

Scoping. The definition and scope of our focus policy system, IncEc, can change significantly when looking at a local, regional, or national scale, which also depends on who you are asking. Working with the policy partner the GM Strategy document was identified as a potential starting point for the mapping workshop. The interview analysis verified that this was a document that was well understood across the organization. Through documentary analysis it was further clarified that the strategy set out explicit priorities, targets and indicators for policy making and thus would allow the team to identified key components for inclusion in the baseline map.

Pre-workshop engagement. Given the limited availability of participants for this workshop (and that spending long periods of time in a videoconference would be draining) we found it helpful to get an impression of key system variables before the workshop through an online poll (Sec. 4.1). This helped us to spend workshop time effectively on revisiting system components and focusing the discussion on the input attributes of links (see Table I). While an online poll supports each participant with a wider perspective of how other people think important IncEc system components would be, it may not necessarily been aligned with individual view of some participants.

In-workshop engagement. Encouraging interactivity in virtual settings can be even more challenging than face-to-face meetings - it is harder to read body language and the flow of conversation is more easily disrupted - it is therefore important to have very clear instructions and prompts for each facilitators, and to pick the right tool to support the workshop activities. To encourage engagement between participants, we built smaller and more diverse groups (20 people including 12 policy officials classified in four working groups) with support from a senior academic advisor (with significant knowledge about IncEc) and a trained facilitator. For encouraging engagement with the map, we used the functionality of Kumu® and developed a color-coded mapping platform with versatile control panel (Fig. 4) to enable participants see the population of the map in real time. Albeit engaging a diverse group with technology is a challenge per se [4], but our experience was quite positive and the majority of participants were appeared to be impressed by getting real-time resolution of IncEc system in progress of the workshop - typically this requires lots of efforts from modelers after the workshop [4][36].

Post-workshop engagement. The workshop output - a digitized map of the IncEc system - is expected to be used for academic modelling purposes as well as future GMCA strategy discussions. The key to this is building cybernetic capabilities that enable individuals to customize the map based on their own priorities and refine it but at the same time encouraging collaborative work by providing a wider whole-system view (to those who have access to the map). We stepped towards this goal by arranging map-sharing sessions, designing an information package and feedback survey, developing master database, and providing access to the map for participants and a wider community who showed interest in using it. In addition to increasing utility of the map, we found this mixed approach helpful in the verification process and revealing the gaps.

Aggregation. The proposed measures of aggregating maps provided a systematic way of identifying areas in the map that participants felt more confident about them, also areas with a better contribution (of different groups), as well as controversial areas where participants raised concerns - while minimizing information loss. Another layer of data aggregation performed pre-workshop in that responses from poll collated to obtain an average importance of system components in the baseline map.

Analysis. Applying network analytics to the aggregated map could provide useful insights on structural dynamics of the IncEc system, in particular, a static model of critical components and pathways across the system - acting as a common ground in future discussions with policy partners. However, whilst the Kumu® provides powerful visualization and flexible data population, it only offers limited analytical capabilities which does not allow for running simulation models. As a result, the outcome of the abstract model was found to be effective for building common ground, conceptual modelling, and expanding understanding across policy areas. 
Therefore, it was suitable for the purpose of this study, but had limited functionality in answering to specific policy scenarios.

Evaluation. Central to the evaluation process in this study has been the need to understand what is a good enough representation? In particular, does the map sufficiently represent what participants expected to see? Does it have the potential to be used by a broader set of audience? How much detail is required to understand how the system functions? While it was generally accepted that the proposed process could successfully develop a common ground for such a complex system, important concerns arose about the tension between granularity of information (required by different user groups) and generality of its use (across the consortium and policy partners). As a result, a narrower scope was agreed for further iteration of the workshop in order to focus on specific policy areas to flesh out the missing details in the first round. The process is agreed to be a series of workshops, 90 minutes in duration, and with a smaller groups of 3-5 topic experts. For future directions, it was also suggested that we study the alignment between evidence that come from policy workshops with relevant evidence in scientific literature.

Potential. Cybernetic participatory mapping is a move towards digitalization of modelling with (and continuous stakeholder engagement), with the goal to not only develop better (more confident) systems maps but also enhancing their utility to inform decision-making. Documented cases of fully virtual PSM in policymaking seem rare, so the authors hope this study makes a valuable contribution to improving our knowledge of the strengths and limitations of online policy systems mapping.

\section{REFERENCES}

[1] M. S. Reed, "Stakeholder participation for environmental management: A literature review," Biol. Conserv., vol. 141, no. 10, pp. 2417-2431, 2008.

[2] A. Voinov and F. Bousquet, "Modelling with stakeholders," Environ. Model. Softw., vol. 25, no. 11, pp. 1268-1281, 2010.

[3] A. Voinov et al., "Modelling with stakeholders - Next generation," Environ. Model. Softw., vol. 77, pp. 196-220, 2016.

[4] B. Wilkerson, A. Aguiar, C. Gkini, I. Czermainski de Oliveira, L. K. Lunde Trellevik, and B. Kopainsky, "Reflections on adapting group model building scripts into online workshops," Syst. Dyn. Rev., pp. $1-15,2020$.

[5] R. P. Kingston, "Public participation in local policy decisionmaking: The role of Web-based mapping," Cartogr. J., vol. 44, no. 2, pp. 138-144, 2007.

[6] P. Meier et al., "The SIPHER consortium: Introducing the new UK hub for systems science in public health and health economic research," Wellcome Open Res., vol. 4, pp. 1-10, 2019.

[7] H. von Foerster, "Cybernetics of Cybernetics," in Understanding Understanding, 2003, pp. 283-286.

[8] GMCA, "Who we are," GMCA, 2019.

https://www.greatermanchester-ca.gov.uk/who-we-are/.

[9] R. Lupton, C. Hughes, A. Macdougall, H. Goldwyn-simpkins, and A. Hjelmskog, "Inclusive Growth in Greater Manchester 2020 and beyond Taking stock and looking forward," 2019.

[10] J. L. A. Geurts and C. Joldersma, "Methodology for participatory policy analysis," Eur. J. Oper. Res., vol. 128, no. 2, pp. 300-310, 2001.

[11] M. Hassannezhad, S. Cassidy, and P. Clarkson, "Connectivity as the capacity to improve an organization's decision-making," in Procedia CIRP, 2019, vol. 84, pp. 231-238.

[12] K. Moon et al., "Mental models for conservation research and practice," Conserv. Lett., no. March 2018, pp. 1-11, 2019.
[13] R. Klimoski and S. Mohammed, "Team Mental Model: Construct or Metaphor?," J. Manage., vol. 20, no. 2, pp. 403-437, 1994.

[14] G. Király, A. Köves, G. Pataki, and G. Kiss, "Assessing the Participatory Potential of Systems Mapping," Syst. Res. Behav. Sci., vol. 33, no. 4, pp. 496-514, 2016.

[15] D. U. Hooper et al., "Effects of biodiversity on ecosystem functioning: a consensus of current knowledge," Ecol. Monogr., vol. 75 , no. 1, pp. 3-35, 2005.

[16] M. Van Der Meer, J. Visser, and T. Wilthagen, "Adaptive and reflexive governance: The limits of organized decentralization," Eur. J. Ind. Relations, vol. 11, no. 3, pp. 347-365, 2005.

[17] S. C. Moser and J. A. Ekstrom, "Taking ownership of climate change: Participatory adaptation planning in two local case studies from California," J. Environ. Stud. Sci., vol. 1, no. 1, pp. 63-74, 2011.

[18] M. Lippe et al., "Building on qualitative datasets and participatory processes to simulate land use change in a mountain watershed of Northwest Vietnam," Environ. Model. Softw., vol. 26, no. 12, pp. 1454-1466, 2011.

[19] R. Hewitt, H. van Delden, and F. Escobar, "Participatory land use modelling, pathways to an integrated approach," Environ. Model. Softw., vol. 52, pp. 149-165, 2014.

[20] S. Gray et al., "Purpose, processes, partnerships, and products: Four Ps to advance participatory socio-environmental modeling: Four," Ecol. Appl., vol. 28, no. 1, pp. 46-61, 2018.

[21] A. Voinov et al., "Tools and methods in participatory modeling: Selecting the right tool for the job," Environ. Model. Softw., vol. 109, no. August, pp. 232-255, 2018.

[22] J. B. McLoughlin and J. N. Webster, "Cybernetic and GeneralSystem Approaches to Urban and Regional Research: A Review of the Literature," Environ. Plan. A Econ. Sp., vol. 2, no. 4, pp. 369408, 1970.

[23] J. Steinbruner, The Cybernetic Theory of Decision: New Dimensions of Political Analysis. Princeton University Press, 2003.

[24] M. L. Cadwallader, "The Cybernetic Analysis of Change in Complex Social Organizations," Am. J. Sociol., vol. 65, no. 2, pp. 154-157, 1959.

[25] M. Sedlacko, A. Martinuzzi, I. Røpke, N. Videira, and P. Antunes, "Participatory systems mapping for sustainable consumption: Discussion of a method promoting systemic insights," Ecol. Econ., vol. 106, pp. 33-43, 2014.

[26] D. Meadows, Thinking in systems: A primer. Chelsea green publishing, 2008.

[27] J. R. Edwards, "A Cybernetic Theory of Stress, Coping, and WellBeing in Organizations," Acad. Manag. Rev., vol. 17, no. 2, pp. 238-274, 1992.

[28] H. Dubberly and P. Pangaro, "Cybernetics and Design: Conversations for Action," Cybern. Hum. Knowing, vol. 22, no. 23, pp. 73-82, 2015.

[29] A. L. Oliver and K. Montgomery, "A system cybernetic approach to the dynamics of individual- and organizational-level trust," Hum. Relations, vol. 54, no. 8, pp. 1045-1063, 2001.

[30] M. Hassannezhad, S. Cassidy, and P. Clarkson, "Predicting Decision Propagation in Complex Sociotechnical Systems," IEEE Syst. J., 2021.

[31] A. S. Penn et al., "Participatory development and analysis of a fuzzy cognitive map of the establishment of a bio-based economy in the Humber region," PLoS One, vol. 8, no. 11, 2013.

[32] R. Lopes and N. Videira, "Modelling feedback processes underpinning management of ecosystem services: The role of participatory systems mapping," Ecosyst. Serv., vol. 28, pp. 28-42, 2017.

[33] S. Mehryar, R. Sliuzas, N. Schwarz, A. Sharifi, and M. van Maarseveen, "From individual Fuzzy Cognitive Maps to Agent Based Models: Modeling multi-factorial and multi-stakeholder decision-making for water scarcity," J. Environ. Manage., vol. 250, no. June 2018, p. 109482, 2019.

[34] P. J. Giabbanelli, S. A. Gray, and P. Aminpour, "Combining fuzzy cognitive maps with agent-based modeling: Frameworks and pitfalls of a powerful hybrid modeling approach to understand humanenvironment interactions," Environ. Model. Softw., vol. 95, pp. 320325, 2017.

[35] H. Herrera and B. Kopainsky, "Using system dynamics to support a participatory assessment of resilience," Environ. Syst. Decis., vol. 40, no. 3, pp. 342-355, 2020. 
[36] L. Freebairn, J. A. Atkinson, N. D. Osgood, P. M. Kelly, G. McDonnell, and L. Rychetnik, "Turning conceptual systems maps into dynamic simulation models: An Australian case study for diabetes in pregnancy," PLoS One, vol. 14, no. 6, pp. 1-27, 2019.

[37] J. Xue, D. Gui, J. Lei, F. Zeng, D. Mao, and Z. Zhang, "Model development of a participatory Bayesian network for coupling ecosystem services into integrated water resources management," $J$. Hydrol., vol. 554, pp. 50-65, 2017.

[38] E. Nabavi, K. A. Daniell, and H. Najafi, "Boundary matters: the potential of system dynamics to support sustainability?," J. Clean. Prod., vol. 140, pp. 312-323, 2017.

[39] P. J. Giabbanelli and M. Baniukiewicz, "Navigating Complex Systems for Policymaking Using Simple Software Tools," in Smart Innovation, Systems and Technologies, vol. 93, 2018, pp. 21-42.

[40] S. Tisue and U. Wilensky, "NetLogo: Design and implementation of a multi-agent modeling environment," Agent 2004 Conf. Soc. Dyn. Interact. Reflexivity Emerg., 2004.

[41] C. M. Burnett, "Incorporating the participatory process in the design of geospatial support tools: Lessons learned from SeaSketch," Environ. Model. Softw., vol. 127, no. January 2019, p. 104678, 2020.

[42] P. Taillandier et al., "Building, composing and experimenting complex spatial models with the GAMA platform," Geoinformatica, vol. 23, no. 2, pp. 299-322, 2019.

[43] A. Singer et al., "Translating community narratives into semiquantitative models to understand the dynamics of socioenvironmental crises," Environ. Model. Softw., vol. 97, pp. 46-55, 2017.

[44] M. van Vliet, K. Kok, and T. Veldkamp, "Linking stakeholders and modellers in scenario studies: The use of Fuzzy Cognitive Maps as a communication and learning tool," Futures, vol. 42, no. 1, pp. 1-14, 2010.

[45] H. S. Firmansyah, S. H. Supangkat, A. A. Arman, and P. J. Giabbanelli, "Identifying the Components and Interrelationships of Smart Cities in Indonesia: Supporting Policymaking via Fuzzy Cognitive Systems," IEEE Access, vol. 7, pp. 46136-46151, 2019.

[46] U. Özesmi and S. L. Özesmi, "Ecological models based on people's knowledge: A multi-step fuzzy cognitive mapping approach," Ecol. Modell., vol. 176, no. 1-2, pp. 43-64, 2004.

[47] S. Mehryar, R. Sliuzas, A. Sharifi, D. Reckien, and M. van Maarseveen, "A structured participatory method to support policy option analysis in a social-ecological system," J. Environ. Manage., vol. 197, pp. 360-372, 2017.

[48] E. I. Papageorgiou and J. L. Salmeron, "A review of fuzzy cognitive maps research during the last decade," IEEE Trans. Fuzzy Syst., vol. 21, no. 1, pp. 66-79, 2013.

[49] E. I. Papageorgiou, "Learning algorithms for fuzzy cognitive maps A review study," IEEE Trans. Syst. Man Cybern. Part C Appl. Rev., vol. 42, no. 2, pp. 150-163, 2012.

[50] G. Felix, G. Nápoles, R. Falcon, W. Froelich, K. Vanhoof, and R. Bello, "A review on methods and software for fuzzy cognitive maps," Artif. Intell. Rev., vol. 52, no. 3, pp. 1707-1737, 2019.

[51] J. Aguilar, "Multilayer Cognitive Maps in the Resolution of Problems using the FCM Designer Tool," Appl. Artif. Intell., vol. 30, no. 7, pp. 720-743, 2016.

[52] V. S. Kireev, I. S. Smirnov, and V. S. Tyunyakov, "Automatic fuzzy cognitive map building online system," in Procedia Computer Science, 2018, vol. 123, pp. 228-233.

[53] G. Nápoles, M. L. Espinosa, I. Grau, and K. Vanhoof, "FCM Expert: Software Tool for Scenario Analysis and Pattern Classification Based on Fuzzy Cognitive Maps," Int. J. Artif. Intell. Tools, vol. 27, no. 7, 2018.

[54] P. J. Giabbanelli, M. Fattoruso, and M. L. Norman, "Cofluences: Simulating the spread of social influences via a hybrid agentbased/fuzzy cognitive maps architecture," SIGSIM-PADS 2019 Proc. 2019 ACM SIGSIM Conf. Princ. Adv. Discret. Simul., no. May, pp. 71-82, 2019.

[55] GMCA, "Our People, Our Place," 2017.

[56] J. P. Carvalho, "On the semantics and the use of fuzzy cognitive maps and dynamic cognitive maps in social sciences," Fuzzy Sets Syst., vol. 214, pp. 6-19, 2013.

[57] P. Hovmand, "Community based system dynamics," Community Based Syst. Dyn., vol. 9781461487, pp. 1-104, 2014.

[58] Y. Iwasaki and H. A. Simon, "Causality and model abstraction Yumi,” Artif. Intell., vol. 67, pp. 143-194, 1994.
P. Clarkson, C. Simons, and C. Eckert, "Predicting Change Propagation in Complex Design," J. Mech. Des. Trans. ASME, vol. 136, no. August 2014, pp. 1-13, 2004.

[60] F. Barzegar, M. Hassannezhad, M. Cantamessa, and P. Clarkson, "On the Functionality of Cambridge Advanced Modeller: A Systematic Qualitative-Quantitative Approach,” 2018. 


\section{Supplementary 1. Input attributes of systems mapping workshop and their color-coding in the mapping platform (used in the workshop to create individual maps)}

\begin{tabular}{|c|c|c|c|}
\hline \multicolumn{2}{|c|}{ Component-related attributes } & \multicolumn{2}{|c|}{ Connection-related attributes } \\
\hline Class & $\begin{array}{ll} & \text { Priority } \\
& \text { Strategy } \\
& \text { Outcome }\end{array}$ & Strength & $\begin{array}{ll}- & \text { Strong (thick grey) } \\
- & \text { Moderate (medium grey) } \\
- & \text { Weak (thin grey) }\end{array}$ \\
\hline Importance & $\begin{array}{ll}\text { Essential } \\
\text { Very important } \\
\text { Important }\end{array}$ & $\begin{array}{l}\text { Confidence } \\
\text { (no change in } \\
\text { color) }\end{array}$ & $\begin{array}{ll}- & \text { Sure } \\
- & \text { Not-sure } \\
\text { - } & \text { Not-defined }\end{array}$ \\
\hline $\begin{array}{l}\text { Source } \\
\text { (no change in } \\
\text { color) }\end{array}$ & $\begin{array}{l}\text { Policy documents } \\
\text { Individual policy interviews } \\
\text { Workshop discussions }\end{array}$ & Directionality & $\begin{array}{ll} & \text { Positive (A+ leads to } \mathrm{B}+) \\
- & \text { Negative (A+ leads to } \mathrm{B}-\mathrm{)} \\
- & \text { Bi-directional (may either ways) }\end{array}$ \\
\hline
\end{tabular}

\section{Supplementary 2. Summary of network centrality analysis for four elicited maps (out of four groups in the workshop) and their unified map}

\begin{tabular}{lccccc}
\hline Criteria & Group A & Group B & Group C & Group D & Unified Map \\
\hline \hline Number of nodes & 59 & 59 & 59 & 59 & 89 \\
Number of links & 187 & 108 & 68 & 128 & 337 \\
Number of links with resolution * & 37 & 11 & 34 & 64 & 194 \\
Fuzziness of the map ** & 0.20 & 0.10 & 0.50 & 0.50 & 0.58 \\
Density of the map & 0.11 & 0.06 & 0.04 & 0.07 & 0.41 \\
Top in-degree component & $\mathrm{O} 3$ & $\mathrm{O} 18$ & $\mathrm{O} 3$ & $\mathrm{O} 3$ & $\mathrm{O} 3$ \\
Top out-degree component & $\mathrm{S} 9$ & $\mathrm{~S} 11$ & $\mathrm{~S} 23$ & $\mathrm{~S} 9$ & $\mathrm{~S} 9$ \\
Number of transmitters & 8 & 18 & 32 & 33 & 2 \\
Number of receivers & 22 & 26 & 24 & 22 & 2 \\
\hline \hline
\end{tabular}

* Links with resolution refers to the links with quality information, the ones for which participants identified the 'connectionrelated attributes': Strength, Directionality, and Confidence. There are many links in the map that, due to time constraints, participants of the workshop could not get back to them and discussing the 'connection-related attributes' further.

** Fuzziness of the map refers to the fraction of the number of links with resolution over the total number of links that identified in group. 
Supplementary 3. Computing the collective confidence in the proposed method (left-hand side) and their frequency in the mapping workshop (right-hand side)

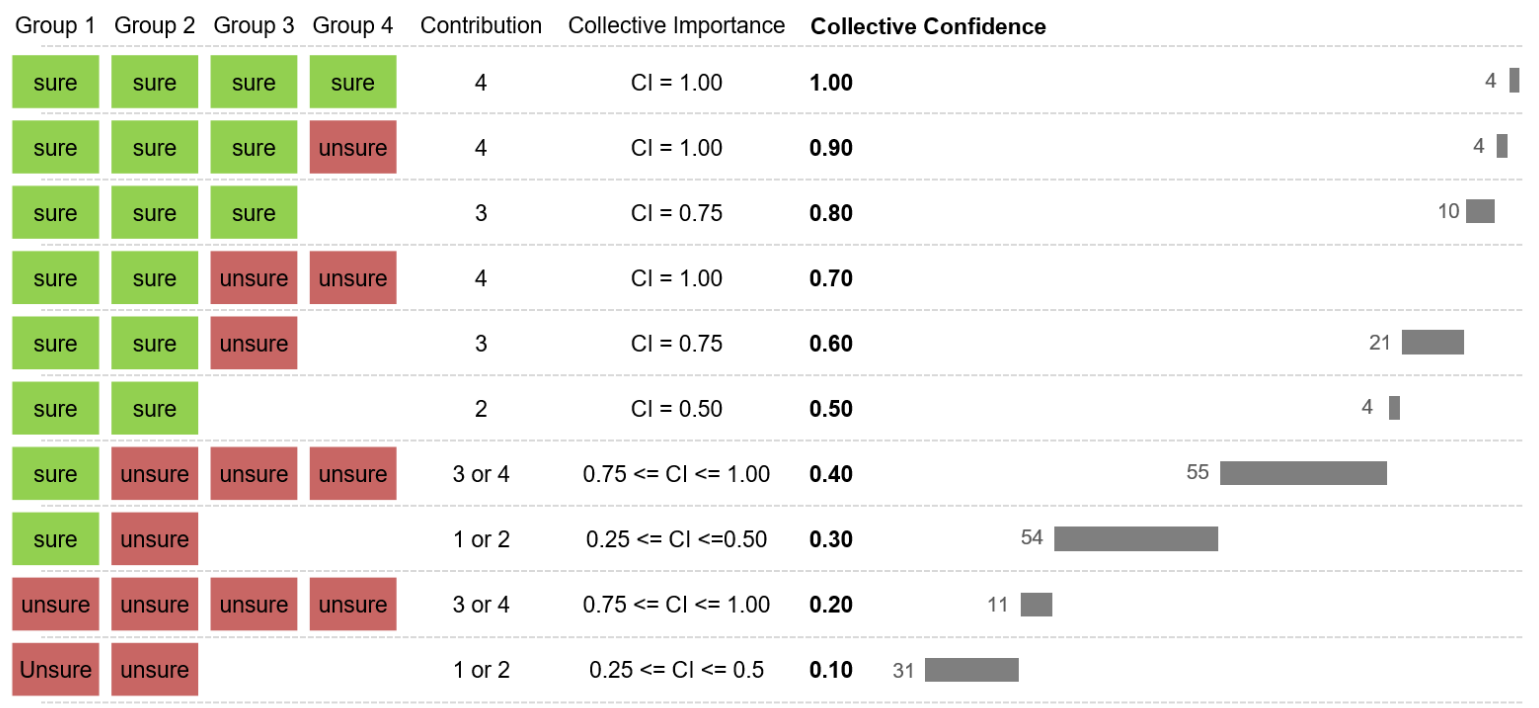


Supplementary 4. Applying degree centrality analysis to the aggregated FCM model

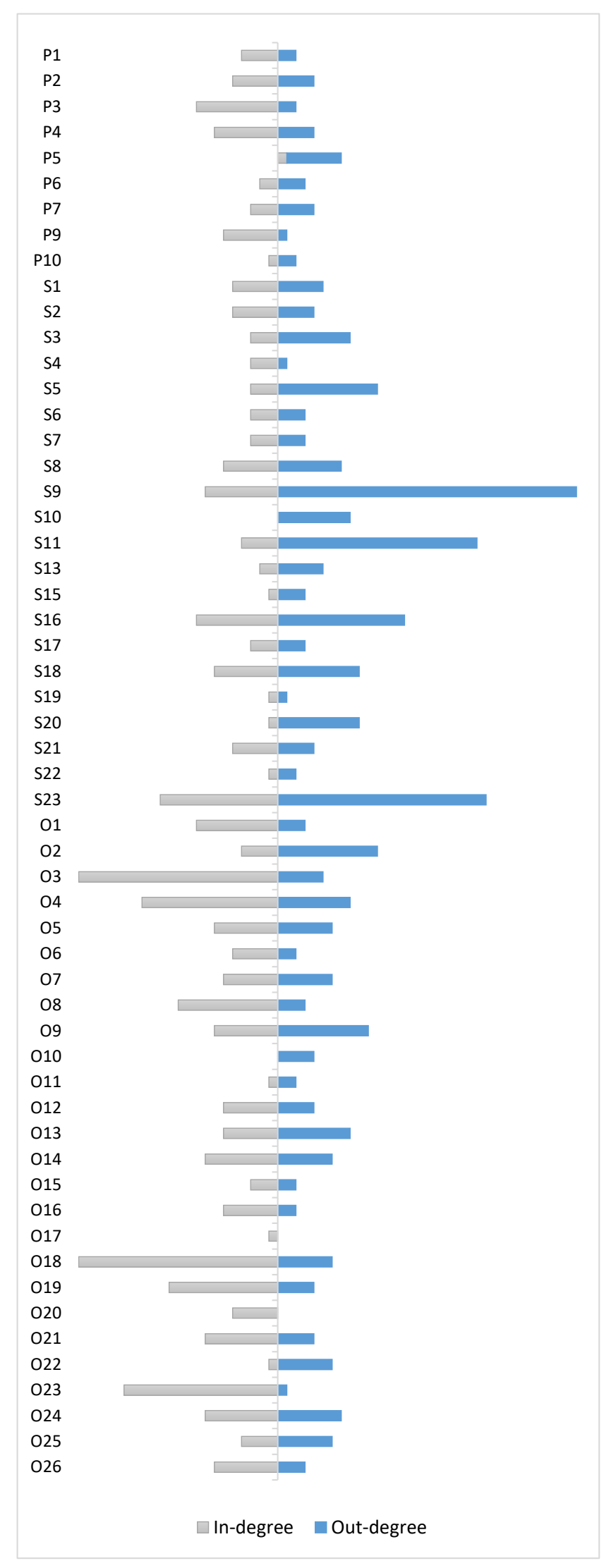


Supplementary 5. Applying change propagation algorithm to the aggregated FCM model: the Risk Portfolio plot (A), Compound Risk plot for $\mathrm{S} 23$ - as one of most influential components (B), and Propagation paths between $\mathrm{O8}$ and $\mathrm{O23}(\mathrm{C})$

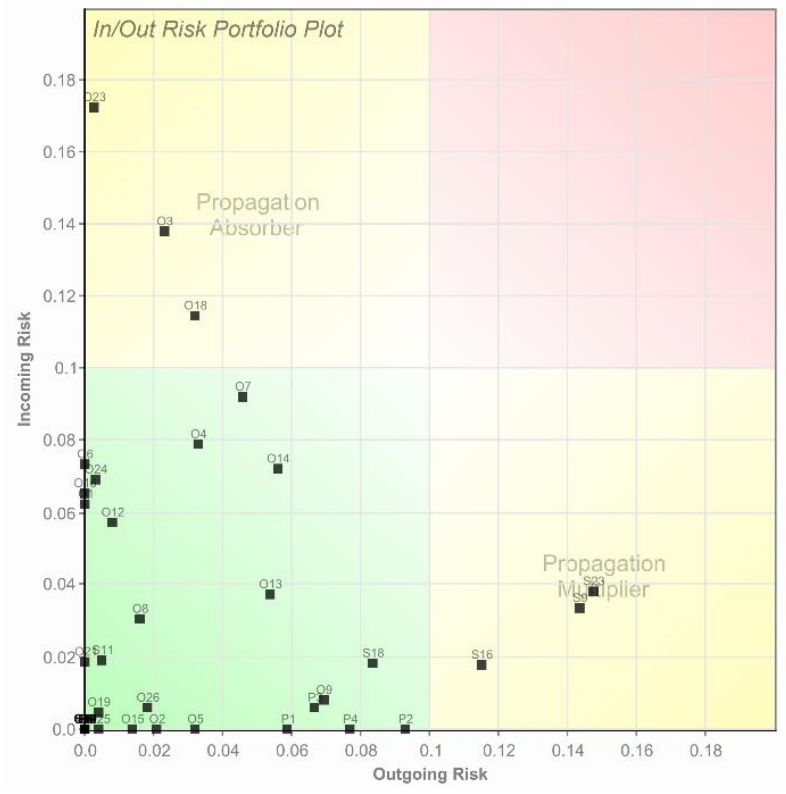

(A) Risk Portfolio plot: showing propagation absorbers (top-left) and multiplier (bottom-right)

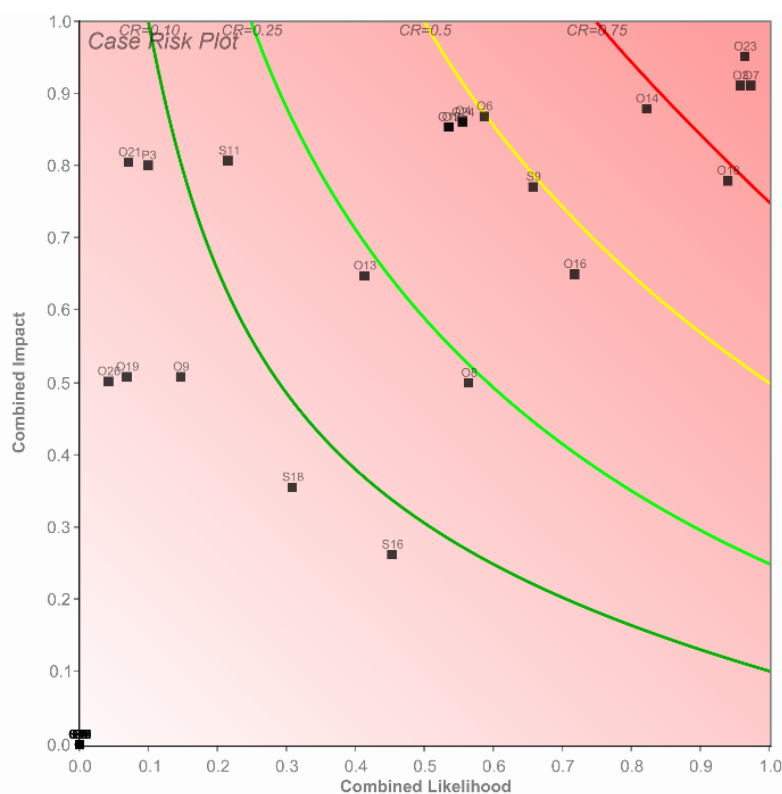

(B) Compound Risk plot for S23 (GM work and skills strategy)

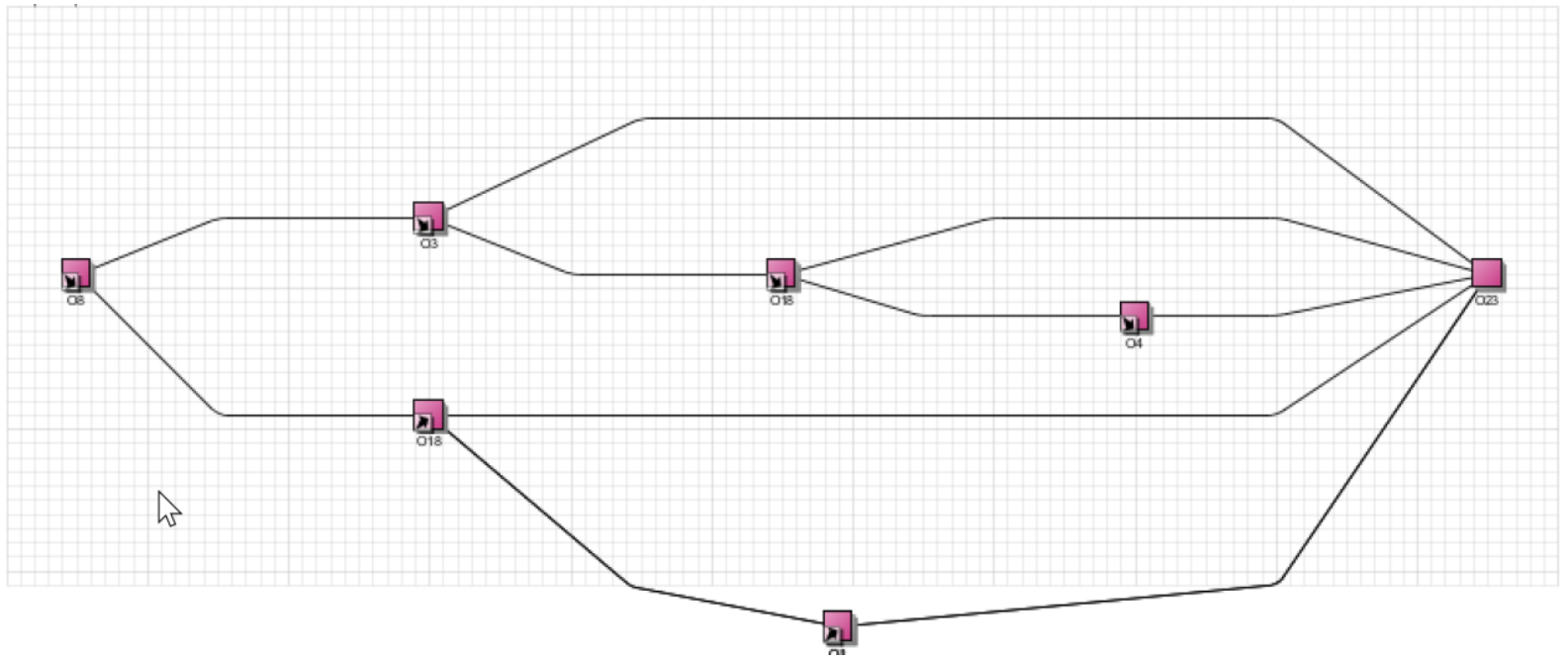

(C) The Propagation paths between Improved Sustainable Production (O8) and Reducing economic inequality $(\mathrm{O} 23)$ shows the indirect influence through their interfacing components O3 (Improved economic growth) and O18 (Increased Sustained Employment) 
Supplementary 6. Dynamic visualization of aggregated map based on centrality network metrics

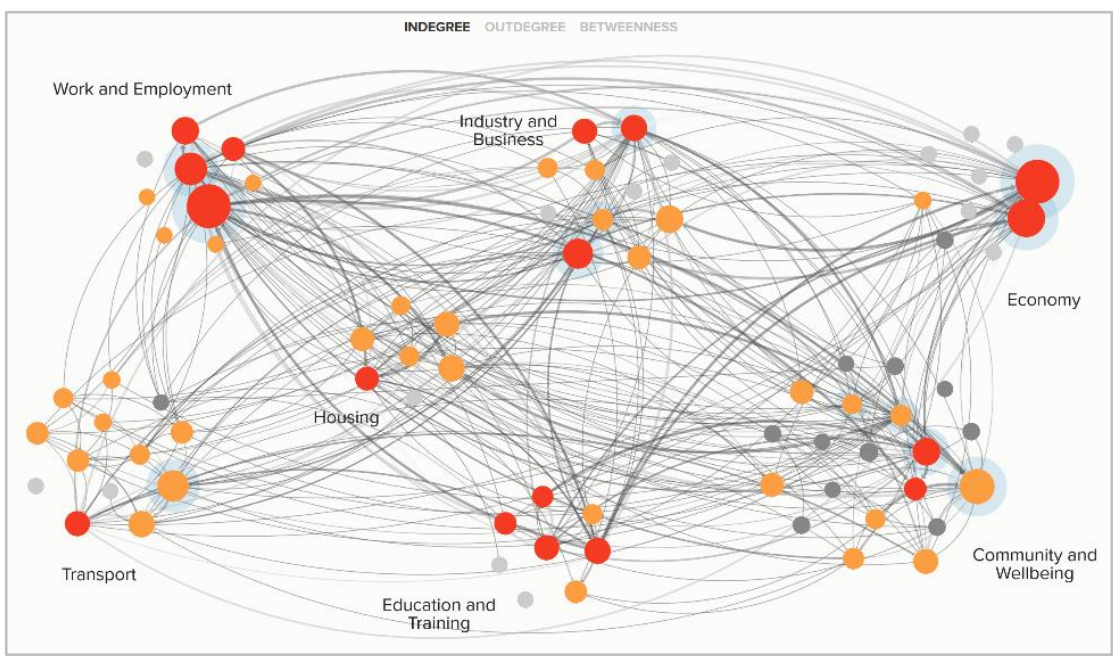

(A) In-degree view: color-coding the map based on the number of inward links

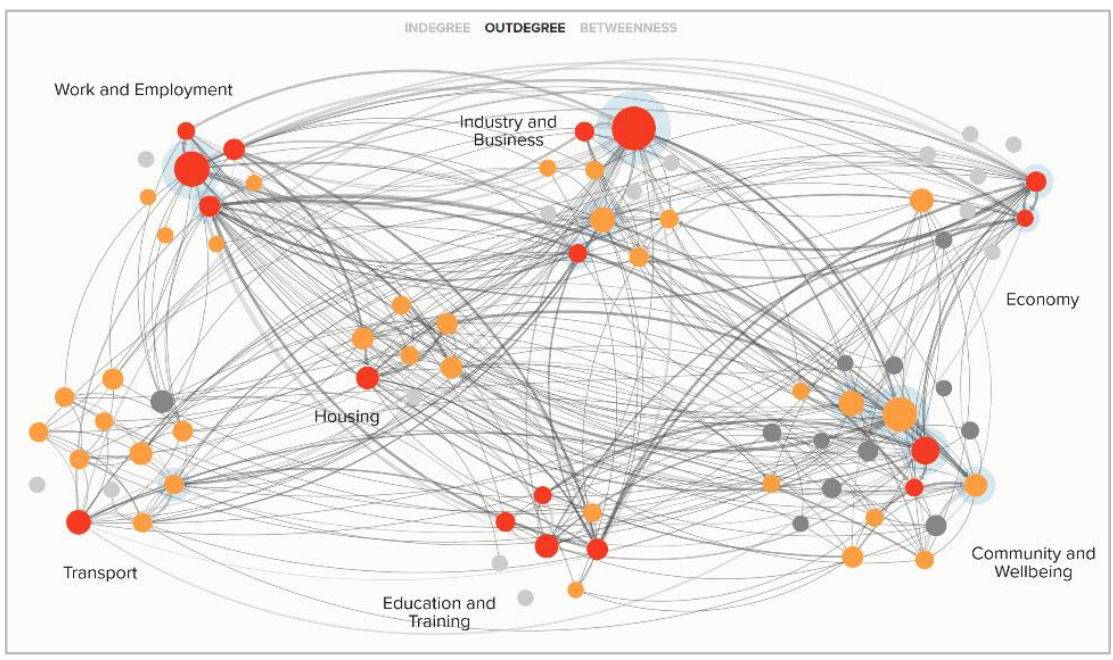

(B) Out-degree view: color-coding the map based on the number of outward links

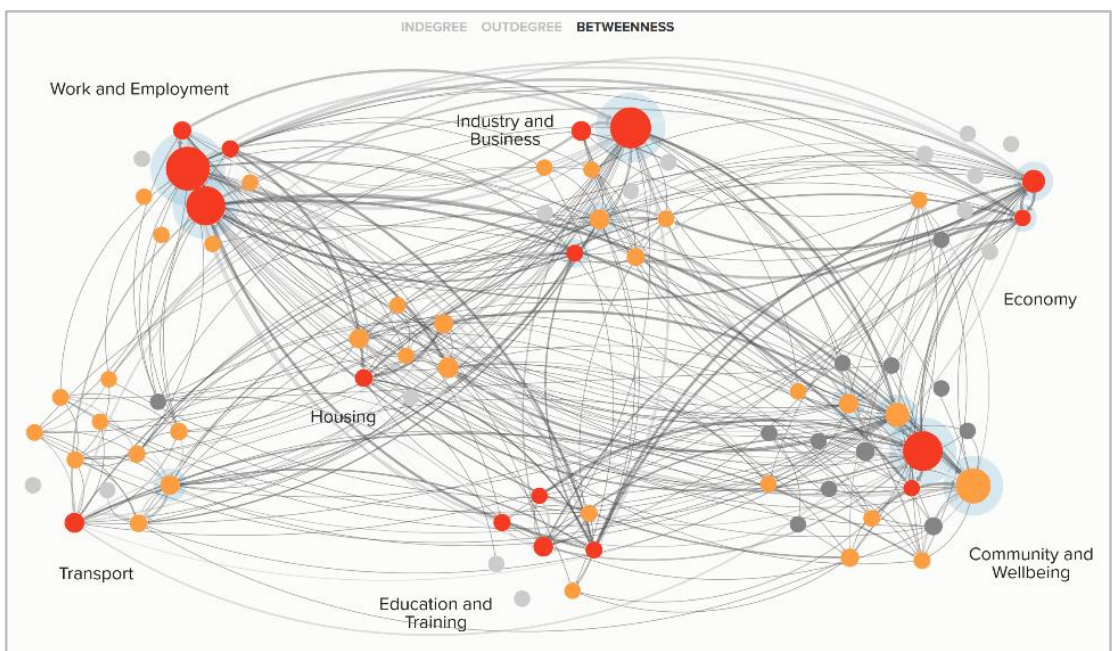

(C) Betweenness view: color-coding the map based on the number of shortest paths passing through a component 